\title{
Immunohistochemical Analysis of Mismatch Repair Deficiency in Colorectal Cancer Patients in Kuantan, Pahang
}

\author{
Muhammad Ishaque F. ${ }^{a, b}$, Asmah Hanim H. ${ }^{a}$, Norlelawati A.T. ${ }^{a}$, Nor Zamzila A. ${ }^{a}$, Feisal E. ${ }^{c}$, \\ Arfahiza S. ${ }^{\text {d }}$ \\ ${ }^{a}$ Department of Pathology and Laboratory, Kulliyyah of Medicine, International Islamic University Malaysia (IIUM), Kuantan, Pahang, Malaysia \\ ${ }^{b}$ Department of Histopathology, Kandahar Medical Faculty, Kandahar University, Durahi, Kandahar, Afghanistan \\ 'Department of Surgery, Kulliyyah of Medicine, International Islamic University Malaysia (IIUM), Kuantan, Pahang, Malaysia \\ ${ }^{\mathrm{d}}$ Department of Pathology, Hospital Tengku Ampuan Afzan Pahang, Kuantan, Pahang, Malaysia
}

\section{ABSTRACT}

INTRODUCTION: The deficient mismatch repair (dMMR) status of colorectal cancer (CRC) is the hallmark of a defective DNA mismatch repair (MMR) system. Immunohistochemistry (IHC) indirectly detects the affected gene by the loss of its protein product. This study aimed to assess the frequency of different types of dMMR status and associate them with the clinicopathological characteristics of CRC patients diagnosed at Hospital Tengku Ampuan Afzan (HTAA) and the Sultan Ahmad Shah Medical Centre (SASMEC) using an immunohistochemical method.

MATERIAL AND METHODS: Formalin-fixed paraffin-embedded (FFPE) tissue blocks of 123 CRC cases were retrieved from the Pathology Department of HTAA and SASMEC for patients diagnosed between 1 January 2017 and 31 December 2018. IHC was performed manually using an Envision Flex Polymer detection kit (Dako) along with four primary anti-mouse antibodies (MLH1, PMS2, MSH2, and MSH6) for the MMR proteins to assess dMMR status. RESULTS: Out of 123 cases, 21 (17.07\%) showed the loss of one or more MMR protein expression and were dMMR. There was no statistically significant association between pMMR (proficient Mismatch repair) and dMMR cases with regards to clinicopathological factors (age, sex, race, site of the tumour, TNM (Tumour Node Metastasis) staging, bowel wall invasion, lymph node metastasis, lymphovascular invasion, histological type, and tumour differentiation). CONCLUSION: IHC is the preferred method and most reproducible test for assessing dMMR status in CRC patients in the histopathology diagnostic laboratory.

KEYWORDS: Colorectal cancer, Immunohistochemistry, MMR, MSI

\section{INTRODUCTION}

Colorectal cancer (CRC) is the second most frequently diagnosed carcinoma in females and the third in males, ${ }^{1}$ and it causes almost 700,000 deaths each year globally. ${ }^{2}$ In Malaysia, CRC is now considered among the most incident cancers in men and placed as the second most frequent cancer in women. ${ }^{3}$

\section{Corresponding Author:}

Asst. Prof. Dr Asmah Hanim Hamdan Department of Pathology and Laboratory Medicine, Kulliyyah of Medicine, International Islamic University Malaysia (IIUM), Bandar Indera Mahkota, Jalan Sultan Ahmad Shah, 25200 Kuantan, Pahang Darul Makmur, Malaysia.

Tel No: +60199112108

Email : drahanim@iium.edu.my
The significance of microsatellite instability (MSI) in CRC has been explored for more than two decades. Microsatellites are simple repeated sequences of DNA, and they are common findings throughout the human genome. ${ }^{4}$ Due to their repetitive nature, microsatellites are sensitive to mismatch errors. Thus, the presence of MSI in CRC is a marker of a dMMR. ${ }^{5,6}$ The presence of MSI indicates an increased rate of a genetic hypermutability condition. Since the discovery of dMMR in familial CRC in the early 1990s, further exposition of the complex mechanisms of dMMR in both the hereditary and sporadic forms of CRC have led to subset characterization of CRC based on distinctive molecular and clinicopathologic features. CRC with a loss of 
expression of $\mathrm{MMR}$ proteins (dMMR tumours) ${ }^{7}$ is present in about $15 \%$ of all CRC cases $(12 \%$ are sporadic, while the remaining 3\% are inherited cases of Lynch Syndrome). ${ }^{8}$ Screening for MMR status in CRC was initially intended to recognise patients with Lynch Syndrome. However, accumulated evidence has suggested that dMMR is a substantial biomarker for the prognosis and treatment prediction of CRC. ${ }^{8}$ Additionally, the immune escape mechanisms recently described in dMMR CRC are a major indicator of the substantial roles of immunotherapy in the treatment of CRC. ${ }^{8-10}$

Based on this new information, testing for MSI or the loss of an MMR protein is currently recommended for most patients with CRC (NCCN, 2014). ${ }^{8,11}$ Of these two methods, MMR protein expression by IHC has the advantage of not requiring additional instruments other than those are already available in a standard histopathology laboratory. Furthermore, the ability to identify the affected gene by detecting the loss of its protein product has a superior advantage over the molecular identification of MSI. The cost of the test is the main hurdle for examining the dMMR system in all cases of CRC and heading the recommendations for CRC patient testing, especially at institutions that are already financially constrained and where the benefit of having a newer therapy for cancer is beyond reach for the majority of patients. An overview of the prevalence of dMMR in CRC cases in a local setting would allow policymakers to plan to offer the service in the near future.

The present study aimed to assess the presence of dMMR in all CRC tissue samples of patients diagnosed at HTAA and the SASMEC in Kuantan for two years. The dMMR status was then associated with all available clinicopathological information. The dMMR status was tested using the IHC method.

\section{METHODS}

\section{Ethical approvals}

The study commenced upon receiving ethical approval from both the IIUM Research Ethics Committee (IREC) of the IIUM (IREC 2019-180) and the Medical Research Ethics Committee (MREC) of the Ministry of Health in Malaysia (NMRR-18-3675-45439).

IMJM Volume 20 No.2, Apr 2021

\section{CRC Cases}

All CRC cases diagnosed between 1 January 2017 and 31 December 2018 at two tertiary hospitals in Kuantan (HTAA and SASMEC) were identified through the laboratory information database. Only cases with available tissue blocks from surgical resection of the large bowel were included in this study. There were 123 CRC cases. The FFPE blocks of these cases were retrieved and subjected to dMMR analysis.

\section{Demographics and clinicopathological data}

The histopathological reports of all cases were reviewed to obtain the following information: age, gender, race, site of the tumour, pTNM (pathological Tumour Node Metastasis) staging, bowel wall invasion, lymph node metastasis, lymphovascular invasion, histological type, and tumour differentiation.

\section{dMMR status assessment}

The dMMR status of the CRC cases was analysed by IHC. Initially, H\&E staining was performed to verify the cellularity of the FFPE tissue blocks. The stained slides were then reviewed by an experienced histopathologist. Immunohistochemical staining was performed manually using a polymer detection kit (Envision FLEX, DAKO, Denmark) with the following reagents: wash buffer $(20 \mathrm{X})$, antigen retrieval solution $(50 \mathrm{X})$, peroxidaseblocking reagent, mouse (linker) for MLH1, HRP (Horseradish peroxidase) and 3.3'-diaminobenzidine tetrahydrochloride chromogen.

The FFPE tissue blocks were sectioned into 3- $\mu \mathrm{m}$ thicknesses for MLH1, MSH2, MSH6, and PMS2 detection. Heat-induced epitope retrieval (HIER) with antigen retrieval solution at a $\mathrm{pH}$ of 9.0 was carried out using a pressure cooker with protocols $97^{\circ} \mathrm{C}$ for $20 \mathrm{~min}$ and at $65^{\circ} \mathrm{C}$ for $10 \mathrm{~min}$ for MLH1, MSH6, and PMS2, and lastly at $121^{\circ} \mathrm{C}$ for $30 \mathrm{sec}$ and then at $95^{\circ} \mathrm{C}$ for $10 \mathrm{sec}$ for MSH2. The tissue sections for MLH1, MSH2, and MSH6 expression were incubated with ready-to-use antimouse primary antibodies for $20 \mathrm{~min}$ each, while the incubation period for PMS2 was 30 min. MMR was considered positive when the tumour's nuclei were stained in the presence of positive internal control. Stromal cells and epithelial cells from the colonic mucosa 
were considered as the internal control. Negative expression was considered when a loss of the tumour cell's nuclear staining was observed despite the presence of positive internal control (Figure 1). Cases that showed positive nuclear staining for all four MMR proteins in the cancerous tissue were termed as pMMR. Cases that demonstrated negative nuclear staining for at least one or more of the MMR proteins were termed as dMMR. ${ }^{2,13}$ This method of reporting has been recommended also by the College of American Pathologists (CAP). ${ }^{14}$

Statistical analyses for the association between dMMR status and clinicopathological data were performed using the Statistical Package for the Social Sciences (SPSS, version 23.0). A chi-square test was used to assess the association of the categorical variables, and $\mathrm{p}<0.05$ was considered statistically significant.

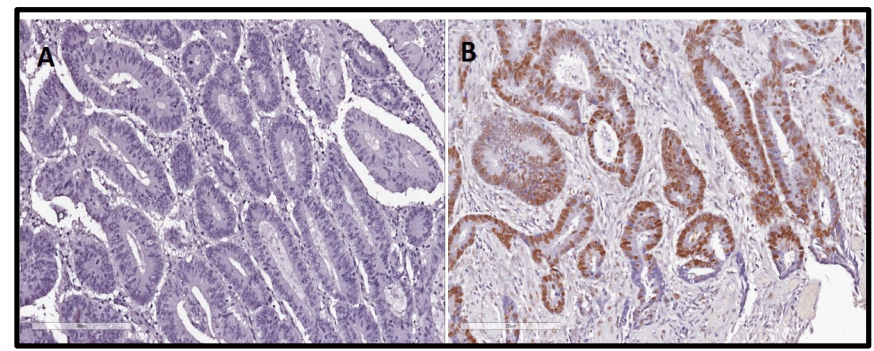

Figure 1: Mismatch repair protein expression (IHC, 200x magnification) A: Negative MMR expression. B: Positive MMR expression.

\section{RESULTS}

\section{Demographic and clinicopathological data}

The mean age of patients in the study was 61.58 (range: 31-86) years. Of the 123 patients, $73(59.35 \%)$ and 50 $(40.65 \%)$ were male and female, respectively. Nineteen $(15.45 \%)$ patients were $\leq 50$ years old, while 104 $(84.55 \%)$ patients were $>50$ years old. Most patients were Malays (90 patients, $73.17 \%$ ), followed by Chinese (30 patients, $24.39 \%$ ), Indians (2 patients, $1.63 \%$ ), and other races (1 patient, $0.81 \%)$. The tumours' characteristics are shown in Table I.

\section{Mismatch repair status}

Out of 123 CRC cases, 102(82.93\%) demonstrated positive nuclear staining for MMR proteins and were
pMMR, while 21(17.07\%) showed a loss of the expression of at least one or more of the MMR proteins and were dMMR. The expression profiles of patients with dMMR are illustrated in Table II. There were no significant associations between MMR status and the demographic and clinicopathological characteristics of the patients (Table III).

Table I: Clinical characteristics of the study sample $(\mathrm{n}=123)$

\begin{tabular}{|c|c|}
\hline Demographic variables & n $(\%)$ \\
\hline \multicolumn{2}{|l|}{ Age } \\
\hline$\leq 50$ & $19(15.45)$ \\
\hline$>50$ & $104(84.55)$ \\
\hline \multicolumn{2}{|l|}{ Sex } \\
\hline Female & $50(40.65)$ \\
\hline Male & $73(59.35)$ \\
\hline \multicolumn{2}{|l|}{ Race } \\
\hline Malay & $90(73.17)$ \\
\hline Chinese & $30(24.39)$ \\
\hline Indian & $2(1.63)$ \\
\hline Indonesian & $1(0.81)$ \\
\hline Clinicopathological data variables & n $(\%)$ \\
\hline \multicolumn{2}{|l|}{ Tumour site } \\
\hline Right-sided & $28(22.76)$ \\
\hline Left-sided & $95(77.24)$ \\
\hline \multicolumn{2}{|l|}{ TNM staging } \\
\hline Stage I & $17(13.82)$ \\
\hline Stage II & $36(29.27)$ \\
\hline Stage III & $65(52.85)$ \\
\hline Stage IV & $5(4.06)$ \\
\hline \multicolumn{2}{|l|}{ Bowel wall invasion } \\
\hline pT1 & $4(3.25)$ \\
\hline pT2 & $26(21.14)$ \\
\hline p'3 & $76(61.79)$ \\
\hline pT4 & $17(13.82)$ \\
\hline \multicolumn{2}{|l|}{ Lymph node metastasis } \\
\hline pNo & $56(45.53)$ \\
\hline pN1 & $44(35.77)$ \\
\hline $\mathrm{pN} 2$ & $22(17.89)$ \\
\hline $\mathrm{pN} 3$ & $1(0.81)$ \\
\hline Lymphovascular invasion & $28(22.76)$ \\
\hline \multicolumn{2}{|l|}{ Histological differentiation } \\
\hline Well differentiated & $20(16.26)$ \\
\hline Moderately differentiated & $100(81.3)$ \\
\hline Poorly differentiated & $3(2.44)$ \\
\hline \multicolumn{2}{|l|}{ Histological type } \\
\hline Mucinous & $20(16.26)$ \\
\hline Non-mucinous & $103(83.74)$ \\
\hline
\end{tabular}


Table II: Details of the immunohistochemistry profiles of the dMMR colorectal cases $(n=21)$

\begin{tabular}{ll}
\hline Types of MMR protein loss & $\mathrm{n}(\%)$ \\
\hline hMLH1 & $1(4.76 \%)$ \\
hMLH1 and hPMS2 & $6(28.57 \%)$ \\
hMLH1, hMSH2 and hPMS2 & $2(9.52 \%)$ \\
hMLH1, hMSH2, hMSH6and hPMS2 & $1(4.76 \%)$ \\
hMSH2 & $4(19.1 \%)$ \\
hMSH2 and hMSH6 & $1(4.76 \%)$ \\
hMSH2 and hPMS2 & $2(9.52 \%)$ \\
hMSH2, hMSH6 and hPMS2 & $1(4.76 \%)$ \\
hPMS2 & $3(14.29 \%)$ \\
\hline
\end{tabular}

\section{DISCUSSION}

The study included 123 CRC cases from two tertiary public hospitals (HTAA and SASMEC) in Kuantan, Pahang. The cases were diagnosed with CRC in 2017 and 2018. Based on data from the Malaysian Statistics Department in 2010, the three main races in Malaysia were Malays (54.7\%), Chinese $(24.6 \%)$, and Indians $(7.3 \%) .{ }^{15}$ However, according to the Malaysian National Cancer Registry, CRC is more prevalent among the Chinese (38.2\%) than in Malays (19.4\%) and Indians $(19 \%) .{ }^{16}$ In the current study, most CRC cases were diagnosed in Malays [ $\mathrm{n}=90$ (73.2\%)], while Chinese patients constituted all but a few of the remaining cases $[\mathrm{n}=30(24.4 \%)]$. The predominant Malay CRC subjects were well correlated with the geographical location of the study samples, where Pahang is a densely Malaypopulated community. The male-to-female ratio of CRC cases in our study was $1.5: 1$, which is similar to the national statistic of 1.3:1.16

Molecular analysis for MSI and IHC for MMR proteins (MLH1, PMS2, MSH2, and MSH6) are the two main methods for determining a patient's MSI or dMMR status. ${ }^{17}$ In this study, an IHC method was used to assess mismatch repair status. This method indirectly specifies the possible gene responsible for the mismatch repair deficiency ${ }^{13,18}$ through an assessment of the corresponding protein products. ${ }^{19}$ As compared to MSI molecular analysis, IHC is an inexpensive and reproducible method $^{7}$ with a highly significant sensitivity $(96.7 \%)$ and specificity $(100 \%) .20,21$

Out of the 123 CRC cases studied, dMMR was identified in $17.1 \%(\mathrm{n}=21)$ [pMMR $(\mathrm{n}=102$ or $82.9 \%)$ ]. The incidence was almost identical to a recently
Table III: The association between the clinicopathological characteristics and the MMR protein expression profile $(n=123)$

\begin{tabular}{|c|c|c|c|}
\hline Variables & $\begin{array}{l}\text { dMMR } \\
(n=21) \\
n(\%)\end{array}$ & $\begin{array}{l}\text { pMMR } \\
(\mathrm{n}=102) \\
\mathrm{n}(\%)\end{array}$ & $p$-value \\
\hline \multicolumn{4}{|l|}{ Age } \\
\hline$\leq 50$ & $4(19.05)$ & $15(14.71)$ & 0.616 \\
\hline$>50$ & $17(80.95)$ & $87(85.29)$ & \\
\hline *Male vs Female & $9(42.86)$ & 41(40.2) & 0.821 \\
\hline \multicolumn{4}{|l|}{ Race } \\
\hline Malay & $15(71.43)$ & $75(73.53)$ & 0.626 \\
\hline Chinese & $5(23.81)$ & $25(24.51)$ & \\
\hline Indian & $1(4.76)$ & $1(0.98)$ & \\
\hline Other & $0(0)$ & $1(0.98)$ & \\
\hline \multicolumn{4}{|l|}{ Site of tumour } \\
\hline Right-sided & $6(28.57)$ & $22(21.57)$ & 0.486 \\
\hline Left-sided & $15(71.43)$ & $80(78.43)$ & \\
\hline \multicolumn{4}{|l|}{$p T N M$ staging } \\
\hline $\mathrm{I}$ & $2(9.52)$ & $15(14.71)$ & 0.564 \\
\hline II & $8(38.1)$ & $28(27.45)$ & \\
\hline III & $11(52.38)$ & $54(52.94)$ & \\
\hline IV & $0(0)$ & $5(4.9)$ & \\
\hline \multicolumn{4}{|c|}{ Bowel wall invasion } \\
\hline pT1 & $0(0)$ & $4(3.92)$ & 0.711 \\
\hline pT2 & $4(19.05)$ & $22(21.57)$ & \\
\hline pT3 & $13(61.9)$ & $63(61.77)$ & \\
\hline p'T4 & $4(19.05)$ & $13(12.74)$ & \\
\hline $\begin{array}{l}\text { Lymph node } \\
\text { metastasis }\end{array}$ & $10(47.62)$ & $46(45.1)$ & 0.833 \\
\hline $\begin{array}{l}\text { Lymphovascular } \\
\text { invasion }\end{array}$ & $4(19.05)$ & $24(23.53)$ & 0.656 \\
\hline \multicolumn{4}{|l|}{ Histological } \\
\hline differentiation & $2(9.5)$ & $18(17.7)$ & 0.515 \\
\hline Well & $18(85.7)$ & $82(80.4)$ & \\
\hline $\begin{array}{l}\text { Moderate } \\
\text { Poor }\end{array}$ & $1(4.8)$ & $2(2.0)$ & \\
\hline $\begin{array}{l}* * \text { Mucinous vs } \\
\text { non-mucinous }\end{array}$ & $5(23.81)$ & $15(14.71)$ & 0.303 \\
\hline
\end{tabular}

Data for *male, ** Mucinous type. p-value is significant at $<0.05$. Chisquare test.

concluded study (17.1\% vs. $16.4 \%)$ predominantly done in Malaysian Chinese subjects. ${ }^{11}$ The incidence rate was also comparable to various published data with a reported dMMR incidence ranging from $10-18 \% .{ }^{11,12 \text {, }}$ 22-26 There was no statistically significant association on the incidence of dMMR status between the different races. The incidence rates of dMMR cases in Malays, Chinese, and Indians were $71.4 \%(n=15), 23.8 \%(n=5)$, and $4.8 \%(\mathrm{n}=1)$, respectively, which follows the study's ethnic distributions. At the same time, the dMMR cases among Malays and Chinese were $16.7 \%$ (15 out of 90) and $16.7 \%$ (5 out of 30 ), respectively. These same types of non-ethnic biases of dMMR status were also reported in other studies done on similar ethnic groups. ${ }^{22,27}$ This finding is in contrast to a significant tendency for 
dMMR CRC among African-Americans compared to Caucasian-Americans. ${ }^{28}$

In our cohort, the most frequently seen dMMR was the concomitant loss of MLH1 and PMS2 in 6 out of 21 cases. This is not a rare finding; many studies have reported similar results. ${ }^{24,25}$ In addition, various combinations of protein losses were discovered in this study ranging from a single protein deletion to the absence of all four proteins (1 case). A literature search revealed only a few reports of patients lacking all four proteins. ${ }^{27}$

This study found no significant association between dMMR status and various clinicopathological characteristics. Although there were reports of a relationship between $\mathrm{dMMR}$ and $\mathrm{CRC}$ in the right colon, ${ }^{12,20,22,29}$ this trend was not observed in the current study. Similarly, the study also did not find any association between dMMR status and lymphovascular invasion, as previously reported. ${ }^{29}$ Many studies are also in agreement with this finding. The inconsistent association between studies may indicate that the association of dMMR with the site of tumour and lymphovascular invasion are incidental findings.

In this study, most dMMR tumours were the nonmucinous type [76.2\% (16 out of 21$]$, which was similar to the findings of a study done by Cheat et $\mathrm{al}^{23}$ on the multiracial Malaysian population. An insignificant association between the dMMR status and the grade of CRC tumour 27,29 and the dMMR status and lymph node metastasis ${ }^{29}$ has also been discovered and explained by others.

\section{CONCLUSION}

This study found a comparable percentage of CRC cases with dMMR status (17\%) as prior studies reported elsewhere. Due to the value of MMR status in the prognostication and management of CRC and the discovery of its relative prevalence here, this study, therefore, proposes that MMR status should be routinely identified in all CRC cases. IHC is known to be sensitive, specific, and reproducible, so this method could be the preferred choice of clinicians in many locations.

\section{CONFLICT OF INTEREST}

There was no conflict of interest among the authors concerning this study.

\section{ACKNOWLEDGEMENT}

This study was financially supported by the International Islamic University of Malaysia (IIUM) and the Ministry of Higher Education in Afghanistan through the Higher Education Development Program. The authors would like to thank the staff of the Pathology and Laboratory Medicine (PALM) department at the IIUM Medical Centre, particularly Br. Mohammad Hanif, Br. Mohammad Amir, and Sr. Nuur Farhana, for their technical support and retrieval of the FFPE blocks.

\section{REFERENCES}

1. Torre, L.A., Bray, F., Siegel, R.L. \& Ferlay, e.a. Global cancer statistics, 2012. CA: a cancer journal for clinicians. 2015; 65, 87-108.

2. Stewart, B.W. \& Wild, C. World Cancer Report 2014. Lyon, France: International Agency for Research on Cancer. World Health Organization, 630 (2014).

3. Van Tze, C.N. et al. Understanding colorectal cancer in Malaysia: A mini-review and pioneering colorectal cancer awareness, screening and treatment project. (2017)

4. Lee, V., Murphy, A., Le, D.T. \& Diaz, L.A. Mismatch repair deficiency and response to immune checkpoint blockade. The oncologist, theoncologist. 2016-0046 (2016).

5. Arzimanoglou, I.I., Gilbert, F. \& Barber, H.R. Microsatellite instability in human solid tumors. Cancer: Interdisciplinary International Journal of the American Cancer Society 82, 1808-1820 (1998).

6. Boland, C.R. \& Goel, A. Microsatellite instability in colorectal cancer. Gastroenterology 138, 2073-2087. e3 (2010).

7. Kawakami, H., Zaanan, A. \& Sinicrope, F.A. Microsatellite instability testing and its role in the management of colorectal cancer. Current treatment options in oncology 16, 30 (2015). 
8. Gupta, R., Sinha, S. \& Paul, R.N. The impact of microsatellite stability status in colorectal cancer. Current problems in cancer (2018).

9. Smyrk, T.C., Watson, P., Kaul, K. \& Lynch, H.T. Tumor-infiltrating lymphocytes are a marker for microsatellite instability in colorectal carcinoma. Cancer 91, 2417-2422 (2001).

10. Le, D.T. et al. PD-1 blockade in tumors with mismatch-repair deficiency. New England Journal of Medicine 372, 2509-2520 (2015).

11. Cheah, P.-L. et al. Screening for microsatellite instability in colorectal carcinoma: practical utility of immunohistochemistry and PCR with fragment analysis in a diagnostic histopathology setting. The Malaysian journal of pathology 41, 91-100 (2019).

12. Seppälä, T. et al. Combination of microsatellite instability and BRAF mutation status for subtyping colorectal cancer. British journal of cancer 112, 1966 (2015).

13. Shia, J. Immunohistochemistry versus microsatellite instability testing for screening colorectal cancer patients at risk for hereditary nonpolyposis colorectal cancer syndrome: part I. The utility of immunohistochemistry. The Journal of molecular diagnostics 10, 293-300 (2008).

14. Bartley, A.N. et al. Template for reporting results of biomarker testing of specimens from patients with carcinoma of the colon and rectum. Archives of Pathology and Laboratory Medicine 138, 166-170 (2014).

15. Malaysia, D.o.S. Population distribution and basic demographic characteristics. Population and Housing Census of Malaysia (2010).

16. Azizah, A.M., Nor Saleha, I., Noor Hashimah, A., Asmah, Z. \& Mastulu, W. Malaysian national cancer registry report 2007-2011. Malaysia cancer statistics, data and figure. Putrajaya: National Cancer Institute, Ministry of Health (2016).

17. Hissong, E., Crowe, E.P., Yantiss, R.K. \& Chen, Y.-T. Assessing colorectal cancer mismatch repair status in the modern era: a survey of current practices and re-evaluation of the role of microsatellite instability testing. Modern Pathology, 1 (2018).

18. Geiersbach, K.B. \& Samowitz, W.S. Microsatellite instability and colorectal cancer. Archives of pathology \& laboratory medicine 135, 1269-1277 (2011).
19. Chen, W., Swanson, B.J. \& Frankel, W.L. Molecular genetics of microsatellite-unstable colorectal cancer for pathologists. Diagnostic pathology 12, 24 (2017).

20. Remo, A., Fassan, M. \& Lanza, G. Immunohistochemical evaluation of mismatch repair proteins in colorectal carcinoma: the AIFEG/GIPAD proposal. Pathologica 108, 104-109 (2016).

21. Wang, T. et al. Immunohistochemical nullphenotype for mismatch repair proteins in colonic carcinoma associated with concurrent MLH1 hypermethylation and MSH2 somatic mutations. Familial cancer 17, 225-228 (2018).

22. Khoo, J.-J., Gunn, A. \& Peh, S.-C. Pattern of hMLH1, hMSH2 and hMSH6 expression and clinical characteristics in a sample of Malaysian colorectal carcinoma cases. Malays J Pathol 35, 45 57 (2013).

23. Cheah, P.-L. et al. Colorectal carcinoma in Malaysians: DNA mismatch repair pattern in a multiethnic population. Asian Pac J Cancer Prev 15, 3287-3291 (2014).

24. Park, J.H. et al. Mismatch repair status in patients with primary operable colorectal cancer: associations with the local and systemic tumour environment. British journal of cancer 114, 562 (2016).

25. Gong, Q. et al. Mismatch repair-deficient status associates with favorable prognosis of Eastern Chinese population with sporadic colorectal cancer. Oncology letters 15, 7007-7013 (2018).

26. Cheah, P.-L. et al. DNA mismatch repair and CD133-marked cancer stem cells in colorectal carcinoma. PeerJ 6, e5530 (2018).

27. Chew, M.-H. et al. Mismatch repair deficiency screening via immunohistochemical staining in young Asians with colorectal cancers. World journal of surgery 37, 2468-2475 (2013).

28. Ashktorab, H. et al. High incidence of microsatellite instability in colorectal cancer from African Americans. Clinical cancer research 9, 11121117 (2003).

29. Korphaisarn, K. et al. Deficient DNA mismatch repair is associated with favorable prognosis in Thai patients with sporadic colorectal cancer. World Journal of Gastroenterology: WJG 21, 926 (2015). 